\title{
Triatominos (Reduviidae: Triatominae) en un foco de enfermedad de Chagas en Talaigua Nuevo (Bolívar, Colombia)
}

\author{
Luis Alberto Cortés ${ }^{1}$, Henry Alberto Suárez ${ }^{2}$ \\ 1 Unidad de Entomología, Secretaría de Salud de Bolívar, Cartagena, Colombia. \\ 2 Bucaramanga, Colombia.
}

Introducción. Se describen las características e importancia epidemiológica de los triatominos presentes en un foco de enfermedad de Chagas en el municipio de Talaigua Nuevo, departamento de Bolívar.

Objetivo. Determinar y correlacionar características ecológicas de los triatominos presentes en un foco de Chagas en el municipio de Talaigua Nuevo, Bolívar.

Materiales y métodos. La captura de los triatominos se realizó en 300 domicilios del casco urbano en los que se revisaron el intra y peridomicilio, así como ambientes extradomésticos (palmas de vino y cúmulos de madera y leña).

Resultados. Se capturaron 103 triatominos pertenecientes a cuatro especies: Triatoma maculata, Eratyrus cuspidatus, Panstrongylus geniculatus y Rhodnius prolixus. T. maculata, fue la especie predominante $(92,3 \%)$. El $93 \%$ de los triatominos fueron capturados en ambientes intradomiciliarios.

Conclusión. Se reporta por primera vez en Bolívar la captura de individuos de T. maculata infectados naturalmente con Trypanosoma sp. Se amplía la distribución geográfica de $P$. geniculatus y $R$. prolixus. El análisis de correspondencias múltiples no encontró ninguna relación significativa entre las características físicas de las viviendas y la presencia de triatominos dentro de ellas.

Palabras clave: insectos vectores, Reduviidae, Triatominae, Triatoma, enfermedad de Chagas.

Triatomines (Reduviidae: Triatominae) in a Chagas disease focus in Talaigua Nuevo (Bolívar, Colombia)

Introduction. The epidemiological importance and ecological characteristics of triatomines were investigated in a Chagas disease focus in Talaigua Nuevo, Bolivar Province.

Objective. To describe and correlate the ecological characteristics of triatomines in a Chagas disease focus.

Materials and methods. Triatomines were collected in 300 domiciles located in the urban zone of Talaigua Nuevo. This included inspection of microhabitats in and around the domestic environment, and also wine palms, woodpiles and firewood.

Results. Among the 103 triatomines collected, four species were identified-- Triatoma maculata, Eratyrus cuspidatus, Panstrongylus geniculatus, and Rhodnius prolixus. T. maculata was the most abundant species (92.23\%). The majority (93\%) were collected in the intradomiciliary environment.

Conclusion. Individuals of T. maculata naturally infected with Trypanosoma were recorded for the first time in Bolivar Province. The distribution range of $P$. geniculatus and $R$. prolixus was expanded. Using a multiple correspondence analysis, no relationship was discerned between the house type and occurrence of triatomines.

Key words: insect vectors, Reduviidae, Triatominae, Triatoma, Chagas disease. 
La tripanosomiasis americana o enfermedad de Chagas es una infección parasitaria crónica causada por el protozoario flagelado Trypanosoma cruzi. El parásito normalmente se transmite al ser humano a través de insectos triatominos estrictamente hematófagos, de la familia Reduviidae, subfamilia Triatominae (1). La subfamilia Triatominae presenta una gran diversidad de especies como producto de su adaptación a ecotopos artificiales y naturales. En Colombia se encuentran 23 especies de triatominos (2) con hábitos silvestres, peridomiciliarios y domiciliarios que les facilitan gran variedad de vertebrados que les sirven como fuente de sangre (3). Sin embargo, las especies domiciliadas de triatominos han recibido el mayor interés debido a su importancia epidemiológica dentro del ciclo de transmisión de la enfermedad de Chagas, situación que ha relegado a segundo plano los estudios de los triatominos con hábitos silvestres (2).

En Colombia existe transmisión natural (vectorial) de $T$. cruzi principalmente en los departamentos de Arauca, Boyacá, Casanare, Cundinamarca, Santander y Norte de Santander y, en menor medida, en Antioquia, Cesar y La Guajira, en el piedemonte de la Sierra Nevada de Santa Marta, Magdalena, Sucre, Meta, Tolima y en las estribaciones de las cordilleras Oriental y Central (4).

Según publicación del Ministerio de Salud, Programa Nacional de Prevención y Control de la Enfermedad de Chagas y la Cardiopatía Infantil, Nodo Occidental, se encontraron tres especies de triatominos en el departamento de Bolívar (5), de las cuales ninguna estaba infectada con $T$. cruzi, y un estudio serológico paralelo no arrojó casos positivos en la prueba de inmunofluorescencia para anticuerpos IgG.

No obstante, en julio de 2003, en Mompós se presentó la muerte de un menor de nueve años con serología positiva para Chagas, y en la misma

\footnotetext{
Correspondencia:

Luis Alberto Cortés Alemán, Unidad de Entomología, Secretaría de Salud de Bolívar, Cartagena centro, calle 36 N7-55 Casa de la Moneda.

Teléfonos: 6648974- fax: 6643600

luisgonatodes@hotmail.com.
}

Recibido:10/06/05; aceptado: 19/09/05 fecha, pero esta vez en zona urbana del municipio de Talaigua Nuevo, se encontraron por tamizaje dos niños menores de cinco años con resultados seropositivos para IFI (Laboratorio de Parasitología Instituto Nacional de Salud de Colombia, INS).

De acuerdo con este cuadro epidemiológico, en este estudio se buscó ampliar los conocimientos acerca del ciclo de transmisión de $T$. cruzi, y se estudiaron características ecológicas de los triatominos domésticos y extradomésticos que allí ocurren, particularmente en relación con las características físicas de las viviendas.

\section{Materiales y métodos}

La investigación se llevó a cabo en el casco urbano del municipio de Talaigua Nuevo, Bolívar, ubicado entre los brazos de Mompós y de Loba del río Magdalena entre $8^{\circ} 48^{\prime}$ y $9^{\circ} 25^{\prime}$ de latitud norte. Esta región corresponde a bosque seco tropical (bs-t) según las zonas de vida de Holdridge (6), con una temperatura promedio anual de $24^{\circ} \mathrm{C}$ y una precipitación que oscila entre $1.500 \mathrm{~mm}$ y $2.000 \mathrm{~mm}$ anuales (7). El municipio cuenta con una población aproximada de 20.000 personas (8) dedicadas sobre todo al cultivo de frutas, pastos y otras actividades como la artesanía y la pesca.

El muestreo entomológico se realizó entre los meses de septiembre y diciembre de 2003, en áreas urbanas, desde las 8:00 hasta las 18:00 horas. El tiempo de captura de los triatominos en una vivienda varió de 20 a 45 minutos; para ello se utilizaron linternas de mano, pinzas largas y recipientes plásticos. Se analizaron 300 domicilios y ambientes extradomésticos (corrales de mamíferos, palomares, nidos de aves, palmeras, palmas de vino y cúmulos de madera o leña) por medio de "muestreo aleatorio simple" (9). Dentro de los domicilios se buscó en todas las habitaciones, muebles y enseres, al igual que en el peridomicilio.

Los insectos recolectados se llevaron al Laboratorio Departamental de Salud Pública de Bolívar, Unidad de Entomología, se identificaron taxonómicamente según claves de Lent y Wigodzinsky (10), y se confirmaron las especies con el Laboratorio de Entomología del INS. Se calcularon los siguientes indicadores entomológicos: índice de infestación (IIV), índice 
de densidades (ID), índice de infección natural (IIN), índice de colonización (IC). La infección por Trypanosoma fue detectada por compresión, la cual consistió en presionar el abdomen de los triatominos capturados y depositar las heces en una lámina porta objeto con solución salina para observación bajo microscopio a 400X. A continuación se muestran las fórmulas usadas en el cálculo de los índices:

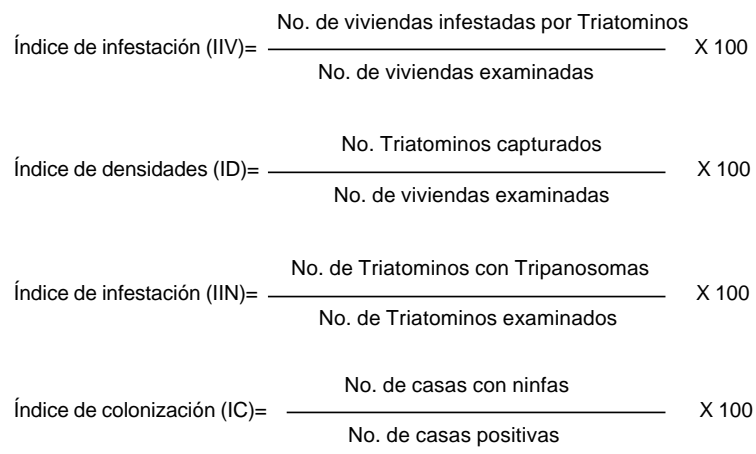

Además, a cada jefe de familia se le hizo una encuesta entomológica de factores de riesgo diseñada por el INS. Con base en ella, se construyó una matriz que consta de doce variables físico-habitacionales de las viviendas y 60 casos positivos para la captura de triatominos (56 casos de T. maculata y cuatro casos de E. cuspidatus); se omitieron los casos positivos de $R$. prolixus y $P$. geniculatus debido a que sólo se presentó uno de cada uno, lo cual impide realizar inferencias estadísticas acerca de estas especies.
Las variables "número de habitantes por vivienda", "años de construida" y "años vividos en la casa" fueron transformadas en categóricas con las clases: "entre 2 y 5 personas, más de 5 personas"; "entre 0 y 10 años de construida, más de 10 años de construida"; "entre 0 y 10 años habitándola"; "más de 10 años habitándola", respectivamente.

Esta matriz se sometió a un análisis de correspondencias múltiples (11) usando Statistica, versión 6, (12) con el fin de evaluar las relaciones existentes entre las características físicohabitacionales y la presencia de las especies mencionadas. Se tomó la variable "especie" como "variable ilustrativa" (es decir, no participó en la construcción de los ejes factoriales), mientras que las demás fueron escogidas como "variables activas" por participar en la construcción de los ejes factoriales. Se tomaron en cuenta los valores propios que estuvieran por encima del promedio.

\section{Resultados}

Se capturaron 103 triatominos pertenecientes a las especies T. maculata $(92,23 \%)$, E. cuspidatus $(5,82 \%), R$. prolixus $(0,98 \%)$ y $P$. geniculatus $(0,98 \%)$. En los ambientes extradomésticos no se encontraron triatominos (cuadro 1).

Del total de triatominos recolectados, 93\% correspondió a capturas en el interior de las viviendas, en las camas y paredes de la sala, mientras que $7 \%$ correspondió a los ambientes peridomiciliarios, principalmente, nidos de palomas y gallinas, y a los patios de las viviendas.

Cuadro 1. Población de triatominos recolectados en el municipio de Talaigua Nuevo, Bolívar.

\begin{tabular}{lcccc}
\hline Ambientes & T. maculata & E. cuspidatus & P. geniculatus & R. prolixus \\
\hline Domésticos & 74 & 3 & 1 & 1 \\
Peridomésticos & 21 & 3 & 0 & 0 \\
Extradomésticos & 0 & 0 & 0 & 0 \\
Total & 95 & $\mathbf{6}$ & $\mathbf{1}$ & $\mathbf{1}$ \\
\hline
\end{tabular}

Cuadro 2. Indicadores entomológicos de los triatominos encontrados en los domicilios de Talaigua Nuevo (Bolívar).

\begin{tabular}{lcccc}
\hline Índices entomológicos & T. maculata & E. cuspidatus & P. geniculatus & R. prolixus \\
\hline IIV & $20,00 \%$ & $2 \%$ & $0,33 \%$ & $0,33 \%$ \\
IDV & $31,66 \%$ & $2 \%$ & - & - \\
IIN & $58,33 \%$ & - & - & - \\
IC & $38,30 \%$ & - & - & - \\
\hline
\end{tabular}


En el cuadro 2 se presentan los indicadores entomológicos para las especies capturadas en Talaigua Nuevo. El índice de infestación de viviendas de $T$. maculata fue superior al de las demás especies encontradas. De los 95 ejemplares de $T$. maculata recolectados, sólo se analizaron por compresión 28 , los cuales dieron un índice de infección natural de $58,33 \%$. De estos 28 individuos, 3 fueron capturados en nidos de paloma y 25 dentro de las viviendas en el dormitorio. Para E. cuspidatus sólo se evaluaron 2 , cuyas heces resultaron negativas para Trypanosoma. Los individuos de $R$. prolixus y $P$. geniculatus se encontraron muertos.

La mayoría de las viviendas visitadas estaban en buenas condiciones. El $65 \%$ de los domicilios tenía paredes de ladrillo totalmente rebocadas y techos de zinc o eternit con pisos de cemento.

En las viviendas infestadas por los triatominos se encontró que $33 \%$ de los techos eran de eternit, $53 \%$ de zinc y $13 \%$ de palma; el $54 \%$ de las viviendas tenía las paredes rebocadas y sólo $20 \%$ tenia gallineros.

El análisis de correspondencias múltiples dio como resultado que los 8 primeros ejes factoriales están asociados a valores propios superiores al promedio $(0,095)$; sin embargo, para este análisis sólo se tomaron en cuenta los tres primeros ejes, cuyos valores propios son: 0,$354386 ; 0,264907$ y 0,194126 , respectivamente, y explican $42,61 \%$ de la inercia.

Las modalidades de mayor contribución a la inercia del primer eje factorial son: "más de 10 años de construida" $(11,53 \%)$ y "más de 10 años de habitada" $(9,51 \%)$ con coordenadas positivas, enfrentadas a las modalidades: "fumigación: programa oficial" (7,58\%) y "pared no rebocada" $(7,57 \%)$, ambas con coordenadas negativas.

Por su parte, las modalidades "pared: lata" $(21,22 \%)$ y "techo de palma" $(20,28 \%)$, con coordenadas positivas, enfrentadas a "sin pared" (5,28\%) y "piso de tierra y cemento" $(5,28 \%)$, con coordenadas negativas, son las cuatro modalidades de mayor contribución a la inercia del segundo eje factorial.

El tercer eje factorial está definido por las modalidades "anexo: gallinero" (12,9\%), con coordenadas positivas, enfrentada a "animales domésticos: no" $(17,4 \%)$, con coordenadas negativas.

En la figura 1, compuesta por los ejes factoriales uno y tres, se puede observar que $T$. maculata y E. cuspidatus no tienen preferencia por un tipo particular de pared,o de techo, o por la presencia de anexos, además de ser indiferentes a la condición de fumigación de la vivienda.

\section{Discusión}

Todas las especies de triatominos se consideran potencialmente vectores de la enfermedad de Chagas; sin embargo, los factores de comportamiento pueden determinar su capacidad vectorial (2,10,13-15). De acuerdo con estas características, para diseñar, programar y desarrollar medidas de control dirigidas a los triatominos es necesario conocer con precisión la identificación correcta de la(s) especie(s) presente(s), su distribución geográfica, el índice tripano-triatomico, el índice de infestación domiciliaria, así como su comportamiento (1). $T$. maculata representó $92,2 \%$ de todos los individuos capturados; esta especie reúne todas los criterios para considerarse un vector eficiente de la enfermedad del Chagas (2), está señalada como vector secundario de la enfermedad en Venezuela (13), y se encuentra en nueve departamentos de Colombia y en tres municipios del departamento de Bolívar $(2,5)$. T. maculata generalmente se encuentra en el peridomicilio (3); sin embargo, en este estudio $T$. maculata mostró un carácter intradoméstico y se le detectó en el peridomicilio en menor proporción. Los resultados de este estudio sugieren que en el municipio de Talaigua Nuevo esta especie presenta hábitos mayormente domésticos, pues $78 \%$ fue capturado dentro de las viviendas, principalmente en las camas, y que, además, todo el ciclo lo realiza en el interior de las viviendas, dado que en el transcurso del estudio se encontraron adultos, huevos, los diversos estados de ninfa, así como exuvias de ninfas y adultos. Esto sugiere que $T$. maculata colonizó ambientes intra y peridomésticos y que puede adaptarse con relativa rapidez a ecotopos artificiales estables cuando sus hábitats naturales son destruidos, situación 


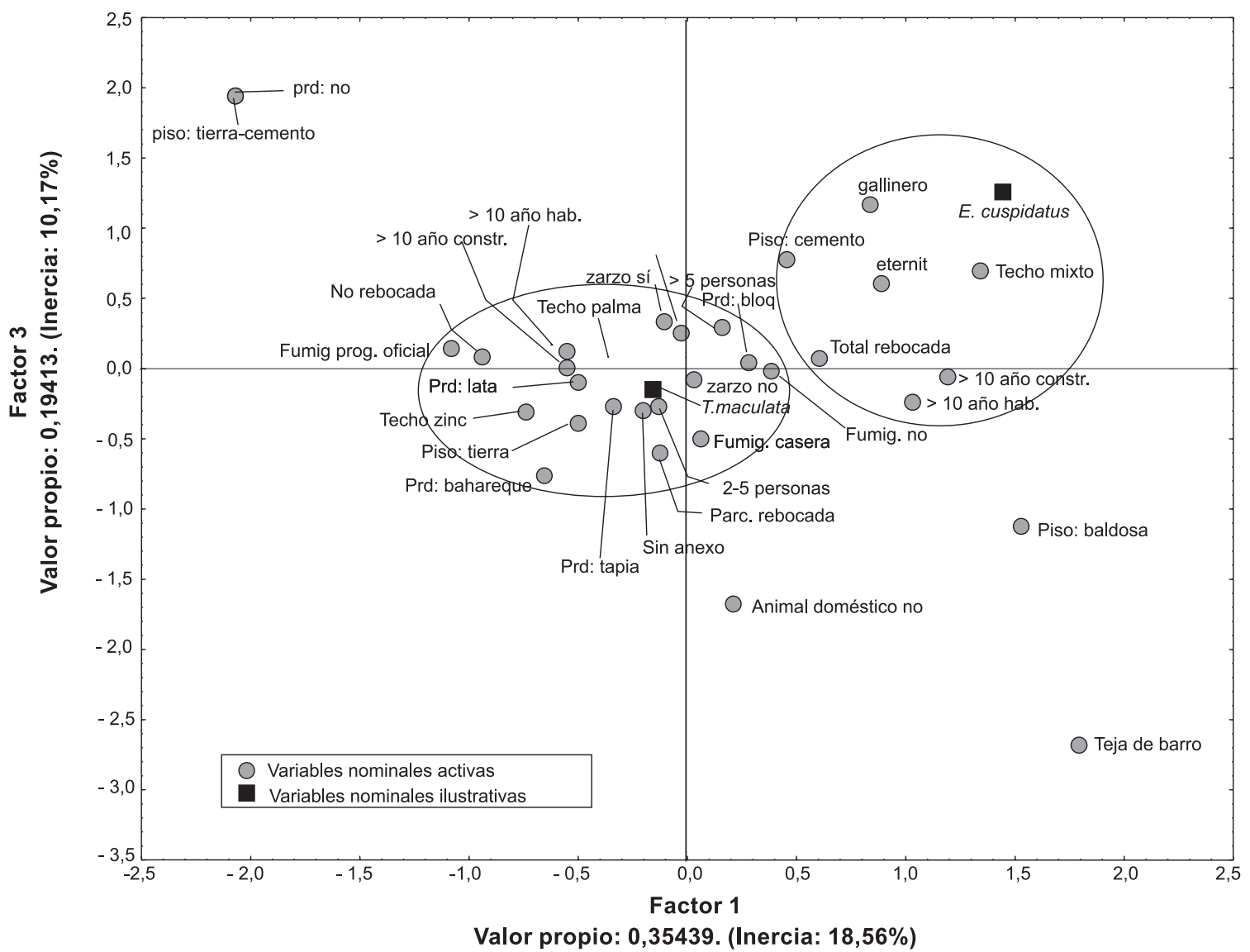

Figura 1. Análisis de correspondencias múltiples de 15 variables y 60 casos positivos para la captura de triatominos en el municipio de Talaigua Nuevo, Bolívar.

que pone en riesgo al municipio de Talaigua Nuevo, dado que se encontró $T$. maculata con un índice de infección natural alto.

$R$. prolixus está catalogada como la principal especie vectora de la enfermedad de Chagas en Colombia, ya que se encuentra en focos domiciliados rurales y urbanos, y en focos silvestres $(16,10)$. Esta especie ha sido registrada en 18 departamentos de Colombia y en un municipio de Bolívar, no infectada por $T$. cruzi (2). R. prolixus no se encontró infectado con $T$. cruzi en Talaigua Nuevo; sin embargo, es necesario seguir vigilando esta especie debido a su importancia epidemiológica. Igualmente debe hacerse con $P$. geniculatus, especie que se considera vectora de la enfermedad de Chagas (2). P. geniculatus se considera silvestre por encontrarse en hábitat de bosque (3) aunque, en algunos casos, presenta domiciliación, como en el caso del municipio de Amalfi, Antioquia (17).

E. cuspidatus se considera como una especie silvestre muy relacionada con nidos de aves, que ocasionalmente se ha localizado en el intradomicilio por su atracción a la luz artificial (18). Los índices entomológicos obtenidos en esta investigación sugieren una infestación reciente; además, la ausencia de huevos, estadios inmaduros, o exuvias en los ambientes donde se recolectaron los adultos sugeriría que $E$. cuspidatus podría colonizar ambientes intra y peridomiciliarios de la localidad estudiada. No obstante, falta hacer un estudio más a fondo de esta especie en la localidad, pues el número de ejemplares recolectados fue bajo. 
Las características de la vivienda, el tipo de material o de construcción parecen no estar relacionados con la invasión de adultos de $E$. cuspidatus ni con la colonización por T. maculata, dado que las viviendas donde se encontraron los vectores en su mayoría están construidas en cemento, con paredes rebocadas, techos de zinc y eternit. Además, las especies E. cuspidatus y $T$. maculata no mostraron afinidad por alguna variable en particular, lo que indica su carácter general.

En conclusión, la especie más abundante fue $T$. maculata. Se reporta por primera vez un triatomino infectado por Trypanosoma sp. en el departamento de Bolívar, y se amplia la distribución geográfica de $R$. prolixus y $P$. geniculatus en el departamento de Bolívar, así como en el país.

Se comprobó la colonización de los ambientes domésticos por $T$. maculata. Por otra parte, la presencia de esta especie en el peridomicilio y en el domicilio demuestra su movilidad entre los distintos ambientes. Si se tiene en cuenta que $T$. maculata colonizó las viviendas, y que los ejemplares recolectados estaban infectados naturalmente en un porcentaje relativamente alto, se puede inferir su participación en la transmisión de T. cruzien el municipio de Talaigua Nuevo.

\section{Agradecimientos}

A Margarita Martínez y Martha Hernández por su apoyo a la logística del estudio; a Alcira Castro por los datos epidemiológicos y a Ramiro Pereira por su apoyo en la toma de muestras y procesamiento en el Laboratorio de Salud Pública Departamental. A Martha Lucía Quiñones por sus sugerencias y por la revisión de este texto. A todos los técnicos del departamento que participaron en la realización de las encuestas. A Víctor Olano y Luis Gualdrón de los Laboratorios de Entomología y de Parasitología del Instituto Nacional de Salud por la confirmación de las especies y de los sueros.

\section{Conflicto de intereses}

Los autores manifiestan que los resultados obtenidos en esta investigación no están relacionados con ningún tipo de intereses.

\section{Financiación}

Este estudio pertenece a los programas de prevención y control de E.T.V. de la Secretaría de Salud de Bolívar, y fue financiado por la Gobernación de Bolívar, Secretaría de Salud.

\section{Referencias}

1. Cáceres AG, Troyes L, González A, Llontop E, Bonilla C, Murias E et al. Enfermedad de Chagas en la región nororiental del Perú. Triatominos (Hemiptera, Heduviidae) presentes en Cajamarca y Amazonas. Rev Per Med Exp Salud Pública 2002;19:17-23.

2. Molina JA, Gualdrón LE, Brochero HL, Olano VA, Barrios D, Guhl F. Distribución actual e importancia epidemiológica de las especies de triatominos (REDUVIIDAE: TRIATOMINAE) en Colombia. Biomédica 2000;20:344-60.

3. Corredor A, Santacruz MM, Páez S, Guateme LA. Distribución de los triatominos domiciliarios en Colombia. Bogota: Instituto Nacional de Salud; 1990. p.1-132.

4. Guhl F, Nicholls S. Manual de procedimientos para el diagnostico de la enfermedad de Chagas. $1^{\underline{a}}$ edición. Santa Fé de Bogotá: Universidad de los Andes; 2001. p.11-2.

5. Restrepo M, Restrepo B, Salazar C, Parra G. Ministerio de la Salud, Programa Nacional de Prevención y Control de la Enfermedad de Chagas y la Cardiopatía Infantil, Nodo Occidental. Instituto Colombiano de Medicina Tropical. Antioquia, Córdoba, y Bolívar. En: Angulo VM, editor. Control y manejo de la tripanosomiasis americana. Bucaramanga: Gráficas Trijaimes; 1999. p.87-93.

6. Holdridge LR, Grenke WC, Hatheway WH, Liang T, Tosi JA. Forest environments in tropical life zones: A pilot study. Oxford: Pergamon Press; 1971.

7. IDEAM. Datos climatológicos de la isla de Mompós, Bolívar. Barranquilla: IDEAM; 2002.

8. DANE. Proyecciones departamentales de población por sexo y edad, Bogota D.C. 1990-2015 estudios censales N2; 2003.

9. Spiegel MR, Stephens LJ. Estadística. Ciudad de México: McGraw Hill Interamericana; 2001. p.541.

10. Lent $\mathbf{H}$, Wygodzinsky P. Revision of the triatominae (Hemiptera, Reduviidae) and their significance as vectors of Chagas disease. Bull Amer Museum Nat Hist 1979;163:123-520.

11. Benzecri, JP. L'analyse des dones. Paris: L'Analyse des correspondences. Dunod; 1973.

12. StatSoft, Inc. STATISTICA (data analysis software system), version 6 2001; www.statsoft.com.

13. Vivas A, Barazarte $\mathbf{H}$, Fernández $\mathbf{D}$. Primer registro de Eratyrus mucronatus Stal, 1959 (Hemiptera. 
Reduviidae) en el ambiente domiciliario en Venezuela. Entomotropica 2001;16:215-7.

14. Zárate L. Comportamiento de los triatomineos en relación a su potencial transmisor de la enfermedad de Chagas (Hemiptera: Reduviidae). Folia Entomológica Mexicana 1984;61:257-71.

15. Carcavallo RU, Galindez I, Jurberg J, Galvao C, Lent $\mathbf{H}$. Pictorial keys for tribes, genera and species of the subfamily Triatominae. En: Carcavallo RU, Galindez I, Jurberg J, Galvao C, Lent H, editors. Atlas of Chagas disease vectors in the Americas. Rio de Janeiro: Editora Fiocruz 1998; p.107-244.
16. Schofield CJ. Triatominae: Biología y control. Reino Unido: Eurocommunica Publications 1994; p.1-79.

17. Wolf M, Arboleda JJ, González C, Manotas LE, Rueda AM. Estudio de tripanosomiasis americana, municipio de Amalfi, vereda Montebello. Boletín Epidemiológico de Antioquia 1994;3:302.

18. Zeledón R, Rabinovich JE. Chagas disease: an ecological appraisal with special emphasis on its insect vectors. Ann Rev Entomol 1981;26:1001-33. 